\title{
Gestational Diabetes - Metabolic Risks of Adult Women With Respect to Birth Weight
}

\author{
D. VEJRAZKOVA ${ }^{1}$, P. LUKASOVA ${ }^{1}$, M. VANKOVA ${ }^{1}$, O. BRADNOVA ${ }^{1}$, G. VACINOVA ${ }^{1}$, \\ J. VCELAK ${ }^{1}$, V. CIRMANOVA ${ }^{1}$, K. ANDELOVA ${ }^{2}$, H. KREJCI ${ }^{3}$, B. BENDLOVA ${ }^{1}$ \\ ${ }^{1}$ Department of Molecular Endocrinology, Institute of Endocrinology, Prague, Czech Republic, \\ ${ }^{2}$ Department of Mother and Child Care, Prague, Czech Republic, ${ }^{3}$ Department of Obstetrics and \\ Gynecology, First Faculty of Medicine, Charles University and General University Hospital in \\ Prague, Czech Republic
}

Received June 2, 2015

Accepted June 16, 2015

\begin{abstract}
Summary
Metabolic disorders such as obesity, insulin resistance and other components of metabolic syndrome (MetS) are connected with birth weight. Low and high birth weight is associated with a higher risk of developing type 2 diabetes mellitus, the mechanism is not clear. In this study, we evaluated the association between birth weight and anthropometric as well as biochemical components of MetS in women with a history of gestational diabetes mellitus (GDM) in comparison with control women. In part of the GDM group, we re-evaluated metabolic changes over 5-8 years. Anthropometry, blood pressure, glucose metabolism during the 3-h oGTT, lipid profile, uric acid, thyroid hormones, and liver enzymes were assessed. From the analyzed components of MetS in adult women we proved the association of low birth weight (birth weight $<25^{\text {th }}$ percentile) with glucose processing, in particular among women with a history of GDM. Low birth weight GDM women revealed significantly higher postchallenge insulin secretion and lower peripheral insulin sensitivity. Re-examinations indicate this association persists long after delivery.
\end{abstract}

\section{Key words}

Gestational diabetes mellitus • Birth weight • Metabolic syndrome - Glucose tolerance

\section{Corresponding author}

D. Vejrazkova, Department of Molecular Endocrinology, Institute of Endocrinology, Narodni 8, 11694 Prague 1, Czech Republic. Fax: +420 224905 325. E-mail: dvejrazkova@endo.cz

\section{Introduction}

Some aspects of our lives are outlined even at the moment of birth. It is well documented that birth weight is associated with metabolic disorders such as insulin resistance, type 2 diabetes mellitus (T2DM), dyslipidemia, and other health problems established as metabolic syndrome (MetS) (Barker et al. 1993, Harder et al. 2007, Whincup et al. 2008). In this respect, unfavorable effect brings both low and high birth weight.

Low birth weight, as a potential reflection of prenatal nutritional restriction, is associated with insulin resistance, T2DM, atherosclerosis, and other MetS symptoms (Hales et al. 1991, Frayling and Hattersley 2001, Harder et al. 2007, Whincup et al. 2008). The mechanism standing behind is not yet fully understood, the most discussed theories are hypothesis of fetal programming (Godfrey and Barker 2000, Meas et al. 2010) and fetal insulin hypothesis (Hattersley et al. 1998, Hattersley and Tooke 1999, Frayling and Hattersley 2001). These theories are not incompatible with each other, conversely, there is a mutual synergy between them. The fetal programming hypothesis suggests that intrauterine lack of nutrients leads to permanent metabolic shift towards insulin resistance. Main purpose of this glucose conserving adaptation is reduction of glucose consumption by periphery in favor of the brain. After postnatal optimization of nutrient intake, rapid weight gain (catch-up-growth) occures, which may predispose these children to central obesity (Ong and Dunger 2000, Ong et al. 2000, Yajnik 2000, Stettler et al. 2002a,b), hypertension (Tzoulaki et al. 2010), and further 
development of insulin resistance (Ibáñez et al. 2003, Durmuş et al. 2010). Fetal insulin hypothesis is based on the assumption that common genetic variants, which lead to moderate decrease in insulin secretion or in insulin function, may predispose to T2DM and, at the same time, they may cause low birth weight, as insulin represents an essential fetal growth factor (Fowden and Forhead 2013). Some studies dealing with monogenic forms of diabetes support this theory. In children, mutations in genes like GCK, INS, INSR, IPF1, ABCC8, HNF1B, or KCNJ11 lead to impaired glucose metabolism and, concurrently, they cause low birth weight (Hattersley et al. 1998, Babenko et al. 2006, Edghill et al. 2006, Slingerland and Hattersley 2006, Støy et al. 2007). However, single gene mutations are too rare to explain the association of T2DM with low birth weight described in population studies.

High birth weight also represents higher risk for T2DM and higher BMI later in life with all related adverse health consequences (Eriksson et al. 2003, Pulizzi et al. 2009). Many studies have demonstrated that higher BMI in childhood often tracks into adolescence and adulthood (Harder et al. 2007, Kitsantas and Gaffney 2010, Oldroyd et al. 2010) and is linked to increased diabetes and MetS risk (Velasquez-Mieyer et al. 2005, Weedon et al. 2006, Freathy et al. 2007, Mohan et al. 2007, Pulizzi et al. 2009, Kalra and Unnikrishnan 2012). Besides, it is assumed that higher T2DM risk in heavy children may reflect diabetogenic potential of the mother in two ways (Dabelea et al. 2000): 1. Maternal fasting glucose concentration is an important determinant of offspring birth weight. Higher maternal glycemia during pregnancy stimulates higher insulin secretion by fetus to maintain normal range of blood glucose. The reason is that maternal insulin, unlike maternal glucose, does not pass the placenta into the fetal circulation because of the molecular size. Considering insulin is in cooperation with IGF I and II a potent fetal growth factor (Fowden and Forhead 2013), higher birth weight is an obvious consequence. 2. Fasting glucose levels and insulin resistance are strongly heritable (Snieder et al. 1999, Freeman et al. 2002, Mills et al. 2004). Thus, half of the genes of predisposed mother passes to the child.

We desided to test association between birth weight and special diabetic condition representing an increased health risk in late adulthood (Mitanchez et al. 2015) - gestational diabetes mellitus (GDM). The aim of our study was to analyze birth weight not only in relation to GDM status, but also with respect to metabolic risk markers of T2DM, insulin resistance, and other components of MetS in adulthood. These markers are anthropometric and physical parameters such as central obesity or hypertension, and biochemical data describing glucose metabolism, lipid profile, and also uric acid (DeBoer et al. 2012). Design of our study allowed us to monitor parameters of glucose metabolism 7 times during the 3-h oGTT and, in part of the GDM group, evaluation of metabolic changes over the years.

\section{Methods}

\section{Study subjects}

Data on birth weight provided 456 adult women. 376 of them were diagnosed with gestational diabetes mellitus (GDM) by the criteria based on the Czech Diabetes Society and the Czech Gynecological and Obstetrical Society (Andělová 2009). All underwent full clinical examination including the 3 -h oGTT. On the day of examination, these women met the 0.5-2 year interval after the delivery. From this cohort of GDM women, 107 underwent complete re-examination after 5-8 years (mean 6.2 \pm 0.47 years), using the same protocol and methods. The control group was composed of 80 fully clinically characterized women who had given birth at least once and had no history of GDM. All participants were without serious pathologies like hormonal disturbances, infections, organ disorders, mental illness etc. Details regarding anthropometric and biochemical data of the repeatedly examined GDM subjects and controls are listed in Table 1. The study protocol was in accordance with institutional ethic guidelines and the national laws and all subjects gave their written informed consent to participate in the study.

\section{Clinical and biochemical characterization}

Body weight, height, and waist, abdominal, as well as hip circumferences were measured in order to calculate body mass index (BMI) and to evaluate body fat distribution by means of waist circumference and waist to hip ratio (WHR).

Venous blood samples were obtained after an overnight fast. Glucose metabolism was characterized by blood glucose (enzymatic reference method with hexokinase; Cobas 6000, Roche Diagnostics, Mannheim, Germany), insulin (ECLIA; Cobas 6000, Roche Diagnostics, Mannheim, Germany), C-peptide (ECLIA; Cobas 6000, Roche Diagnostics, Mannheim, Germany), proinsulin (ELISA, DRG Instruments GmbH, Germany), and glucagon (EURIA-Glucagon Radioimmunoassay, Euro Diagnostica AB, Sweden). 
Table 1. Clinical characteristics of the repeatedly examined GDM subjects and controls.

\begin{tabular}{|c|c|c|c|c|c|}
\hline & $\begin{array}{c}\text { GDM } \\
\mathbf{1}^{\text {st }} \text { examination }\end{array}$ & $\begin{array}{c}\text { GDM } \\
\text { re-examination }\end{array}$ & $\begin{array}{c}\text { p } \\
1^{\text {st }} \text { exam vs. } \\
\text { re-exam } \\
\text { GDM }\end{array}$ & Controls & $\begin{array}{c}\text { p } \\
\text { re-exam } \\
\text { GDM vs. } \\
\text { controls }\end{array}$ \\
\hline$n$ & 107 & 107 & - & 80 & - \\
\hline Age (years) & $33.1 \pm 4.23$ & $40.7 \pm 5.38$ & - & $42.2 \pm 9.29$ & 0.57 \\
\hline \multicolumn{6}{|l|}{ Anthropometry } \\
\hline Systolic blood pressure & $112.2 \pm 14.25$ & $113.4 \pm 15.6$ & 0.51 & $117.8 \pm 14.81$ & 0.06 \\
\hline Diastolic blood pressure & $71.3 \pm 10.52$ & $71.6 \pm 11.25$ & 0.89 & $75.2 \pm 10.03$ & 0.03 \\
\hline$B M I\left(\mathrm{~kg} / \mathrm{m}^{2}\right)$ & $23.7 \pm 4.06$ & $24.8 \pm 4.60$ & 0.08 & $24.7 \pm 4.74$ & 0.75 \\
\hline Waist circumference (cm) & $76.6 \pm 8.73$ & $79.9 \pm 10.66$ & 0.03 & $77.8 \pm 10.46$ & 0.15 \\
\hline WHR & $0.77 \pm 0.053$ & $0.78 \pm 0.067$ & 0.47 & $0.76 \pm 0.055$ & 0.008 \\
\hline Abdominal circumference $(\mathrm{cm})$ & $84.6 \pm 9.10$ & $90.0 \pm 10.88$ & $<0.001$ & $86.3 \pm 11.63$ & 0.008 \\
\hline Gluteal circumference (cm) & $98.7 \pm 7.95$ & $101.9 \pm 9.50$ & 0.016 & $102.2 \pm 9.71$ & 0.87 \\
\hline \multicolumn{6}{|l|}{ Biochemistry } \\
\hline Basal glycemia $(\mathrm{mmol} / \mathrm{l})$ & $4.71 \pm 0.441$ & $5.03 \pm 0.507$ & $<0.001$ & $4.85 \pm 0.64$ & $<0.001$ \\
\hline$A U C_{G l c 7}\left(\mathrm{mmol}^{*} \min / \mathrm{l}\right)$ & $1011 \pm 147.4$ & $1120 \pm 199.6$ & $<0.001$ & $1050 \pm 263.2$ & $<0.001$ \\
\hline Basal insulinemia (mIU/l) & $7.33 \pm 9.312$ & $7.68 \pm 6.367$ & 0.021 & $7.37 \pm 5.369$ & 0.21 \\
\hline$A U C_{\text {Ins } 7}\left(m I U^{*} \min / l\right)$ & $5773 \pm 2658.4$ & $6543 \pm 2771.9$ & 0.024 & $6237 \pm 4755.9$ & 0.008 \\
\hline Basal C-peptide (nmol/l) & $0.64 \pm 0.254$ & $0.64 \pm 0.222$ & 0.64 & $0.61 \pm 0.264$ & 0.09 \\
\hline$A U C_{C-p e p 7}\left(\mathrm{nmol}^{*} \min / \mathrm{l}\right)$ & $371.5 \pm 102.28$ & $355.2 \pm 86.00$ & 0.45 & $381.8 \pm 132.29$ & 0.47 \\
\hline HOMAF & $125.2 \pm 125.51$ & $107.6 \pm 97.12$ & 0.26 & $120.2 \pm 89.35$ & 0.29 \\
\hline HOMAR & $1.57 \pm 2.093$ & $1.78 \pm 1.722$ & 0.005 & $1.66 \pm 1.449$ & 0.09 \\
\hline IS-Matsuda & $8.7 \pm 4.12$ & $7.3 \pm 4.64$ & 0.003 & $8.7 \pm 4.33$ & 0.005 \\
\hline IS-Cederholm & $71.1 \pm 15.02$ & $62.5 \pm 15.52$ & $<0.001$ & $72.2 \pm 22.26$ & 0.003 \\
\hline Cholesterol (mmol/l) & $4.6 \pm 0.79$ & $4.8 \pm 0.77$ & 0.006 & $4.8 \pm 0.77$ & 0.54 \\
\hline HDL (mmol/l) & $1.6 \pm 0.39$ & $1.6 \pm 0.34$ & 0.21 & $1.5 \pm 0.33$ & 0.44 \\
\hline LDL (mmol/l) & $2.5 \pm 0.62$ & $2.7 \pm 0.72$ & 0.035 & $2.8 \pm 0.74$ & 0.42 \\
\hline$T A G(\mathrm{mmol} / \mathrm{l})$ & $0.80 \pm 0.446$ & $0.95 \pm 0.489$ & 0.003 & $1.05 \pm 0.51$ & 0.12 \\
\hline Uric acid (umol/l) & $243.9 \pm 52.80$ & $246.3 \pm 48.54$ & 0.89 & $238.6 \pm 59.02$ & 0.32 \\
\hline$A L T(u k a t / l)$ & $0.32 \pm 0.170$ & $0.31 \pm 0.153$ & 0.88 & $0.36 \pm 0.23$ & 0.05 \\
\hline AST (ukat/l) & $0.39 \pm 0.330$ & $0.32 \pm 0.065$ & 0.29 & $0.39 \pm 0.160$ & 0.017 \\
\hline GGT (ukat/l) & $0.23 \pm 0.133$ & $0.25 \pm 0.136$ & 0.12 & $0.31 \pm 0.286$ & 0.10 \\
\hline TSH (mIU/l) & $2.5 \pm 1.94$ & $2.6 \pm 1.24$ & 0.07 & $2.5 \pm 1.56$ & 0.12 \\
\hline fT3 (pmol/l) & $5.0 \pm 0.76$ & $4.6 \pm 0.66$ & $<0.001$ & $5.0 \pm 1.48$ & 0.04 \\
\hline fT4 (pmol/l) & $15.5 \pm 2.88$ & $15.1 \pm 2.25$ & 0.11 & $15.6 \pm 3.30$ & 0.38 \\
\hline
\end{tabular}

Values are given as mean $\pm S D$, p-level according to Mann-Whitney test.

A 3-h oral glucose tolerance test (oGTT) with $75 \mathrm{~g}$ of glucose load was performed with sampling 7 times in $0,30,60,90,120,150$, and $180 \mathrm{~min}$. Areas under the 7-point oGTT glycemic $\left(\mathrm{AUC}_{\mathrm{Glc}}\right), \mathrm{C}$-peptide $\left(\mathrm{AUC}_{\mathrm{C} \text {-pep7 }}\right)$ and insulin curves $\left(\mathrm{AUC}_{\mathrm{I} 7}\right)$ were calculated. Lipid profile was assessed by total cholesterol (enzymatic colorimetric test; Cobas 6000, Roche Diagnostics, Mannheim, Germany), high density lipoprotein (HDL) (homogeneous enzymatic colorimetric test; Cobas 6000, Roche Diagnostics, Mannheim, Germany), low density lipoprotein (LDL) (homogeneous enzymatic colorimetric test; Cobas 6000, Roche Diagnostics, Mannheim, Germany), and triacylglycerol (TAG) concentrations (enzymatic colorimetric test; Cobas 6000, Roche Diagnostics, Mannheim, Germany). To assess insulin sensitivity, the following three indices were calculated: 
homeostasis model HOMAR $=$ insulin $_{0 \min }[\mathrm{mIU} / \mathrm{l}] \quad \mathrm{x}$ glucose $_{0 \min }[\mathrm{mmol} / \mathrm{l}] / 22.5$, Matsuda index $=104 / \sqrt{ }($ mean insulin $_{0 \text { to120min }}[\mathrm{mIU} / \mathrm{l}] \mathrm{x}$ mean glucose $\mathrm{tto120 \operatorname {min }}[\mathrm{mmol} / \mathrm{l}] \mathrm{x}$ glucose $_{0 \min }[\mathrm{mmol} / \mathrm{l}] \quad \mathrm{x} \quad$ insulin $\left._{0 \min }[\mathrm{mIU} / \mathrm{l}]\right)$, and Cederholm index $=\left[75000+\left(\right.\right.$ glucose $_{0 \min }[\mathrm{mmol} / \mathrm{l}]-$ glucose $_{120 \mathrm{~min}}[\mathrm{mmol} / \mathrm{l}] \times 1.15 \times 180 \times 0.19 \times$ body weight $[\mathrm{kg}]] /\left[\begin{array}{llllll}120 & \mathrm{x} & \text { mean } & \text { glucose } & \\ 0 \text { to120min } & {[\mathrm{mmol} / \mathrm{l}]} & \mathrm{x} & \log \end{array}\right.$ (mean insulin to120min $)$. Beta-cell function was evaluated by $\mathrm{HOMAF}=20 \mathrm{x} \quad$ insulin $_{0 \min }[\mathrm{mIU} / \mathrm{l}] /$ glucose $_{0 \mathrm{~min}}$ $[\mathrm{mmol} / \mathrm{l}]-3.5)$.

Systolic and diastolic blood pressure $[\mathrm{mm} \mathrm{Hg}]$ was determined in a rest state. Furthermore, serum uric acid was assessed photometrically (enzymatic colorimetric test; Cobas 6000, Roche Diagnostics, Mannheim, Germany).

In addition, thyroid hormones TSH, fT3, fT4 (ECLIA; Cobas 6000, Roche Diagnostics, Mannheim, Germany) and liver enzymes ALT (IFCC method with alanine and pyridoxalphosphate; Cobas 6000, Roche Diagnostics, Mannheim, Germany), AST (method with aspartate and pyridoxalphosphate; Cobas 6000, Roche Diagnostics, Mannheim, Germany), and GGT (enzymatic colorimetric test; Cobas 6000, Roche Diagnostics, Mannheim, Germany) were evaluated.

Complete records of questionnaires monitoring anamnestic data regarding birth weight and prematurity information were collected from all our participants. Individuals from multiple pregnancies were excluded from the analysis. Participants born prematurely (GDM: $n=23$, controls: $n=2$ ) were included, however, the presented data directly linked to birth weight were verified also without pre-term born participants, their exclusion had no significant effect on the results. In our study, low birth weight subjects were those below $25^{\text {th }}$ percentile determined from the whole cohort (i.e. GDM merged together with controls), which was equivalent to $2900 \mathrm{~g}$. High birth weight women were those above $75^{\text {th }}$ percentile, equivalent to $3500 \mathrm{~g}$.

\section{Statistical analysis}

Differences in biochemical and anthropometric data between the compared groups were tested by nonparametric Mann-Whitney test owing to the non-normal data distribution (NCSS 2004). Chi-squared test was used to compare quartile distribution of birth weight between GDM and controls. Non-parametric Spearman correlation matrix was applied to assess relations between continuous variables. For comparison of metabolic changes over the years, parametric repeated measures ANOVA (NCSS
2004 and Statgraphics Centurion XVI 16.0.07 software) was applied after power transformation of data. The p-values $<0.05$ (two tailed) were considered to be significant.

\section{Results}

\section{Comparison of GDM and control group}

Anthropometry: Mean birth weight did not differ significantly between the GDM and control group (GDM $3245.5 \pm 478.47$ vs. C $3264.3 \pm 530.14 \mathrm{~g}, \mathrm{p}=0.93$ ). The frequency of subjects with low $(<2900 \mathrm{~g})$ and high $(>3500 \mathrm{~g})$ birth weight was not significantly different between GDM and control group $\left(\mathrm{Chi}^{2}=0.11, \mathrm{p}=0.95\right)$. Anthropometric differences were compared between repeatedly examined GDM subjects and control group. These two cohorts did not differ in age and women in both cohorts were not shortly after delivery, so that anthropometric data are not affected by pregnancy. Results are listed in Table 1. In spite of comparable BMI, GDM women had significantly higher WHR and abdominal circumference, which suggests inclination towards more central fat distribution. This observation is in accordance with higher T2DM risk in women with positive history of GDM.

Biochemistry: Higher basal glycemia as well as higher postchallenge glucose levels expressed as $\mathrm{AUC}_{\mathrm{Glc} 7}$ were observed in GDM group, see all details of the compared parameters in Table 1. Also postchallenge insulin levels expressed as $\mathrm{AUC}_{\mathrm{I} 7}$ were higher in women with positive GDM history. According to Matsuda and Cederholm indices, peripheral insulin sensitivity was lower. Statistical differences between the two groups were observed also in hepatic enzyme AST, thyroid hormone fT3, and diastolic blood pressure, however, these differences were not of clinical relevance.

\section{Comparison of 1 st examination and re-examination of GDM subjects}

Clinical characteristics of re-examined GDM subjects are shown in Table 1. If we focus on the symptoms of MetS, in addition to significant increase in all measured body circumferences, quite complex deterioration was observed in glucose metabolism, especially with respect to basal and stimulated glucose concentrations and indices of insulin sensitivity. Unfavorable changes were detected also in lipid profile, but not in blood pressure or in uric acid concentrations. 
Table 2. Differences between birth weight categories in GDM subjects.

\begin{tabular}{|c|c|c|c|c|c|}
\hline & $\begin{array}{l}\text { 1. quartile } \\
\leq 2900 \mathrm{~g}\end{array}$ & $\begin{array}{c}\text { 2. + 3. quartile } \\
>2900-\leq 3500 \mathrm{~g}\end{array}$ & $\begin{array}{l}\text { 4. quartile } \\
>3500 \mathrm{~g}\end{array}$ & $\begin{array}{c}\text { p } \\
\text { 1. vs. } 2 .+3 . \\
\text { quartile }\end{array}$ & $\begin{array}{c}\text { p } \\
\text { 1. vs. } 4 . \\
\text { quartile }\end{array}$ \\
\hline$n(\Sigma=376)$ & 91 & 195 & 90 & - & - \\
\hline Age (years) & $34.6 \pm 5.08$ & $33.9 \pm 4.90$ & $33.4 \pm 3.74$ & 0.22 & 0.11 \\
\hline \multicolumn{6}{|l|}{ Anthropometry } \\
\hline Systolic blood pressure & $113.8 \pm 13.86$ & $111.3 \pm 12.87$ & $112.0 \pm 11.59$ & 0.27 & 0.40 \\
\hline Diastolic blood pressure & $72.9 \pm 10.67$ & $70.6 \pm 8.79$ & $71.5 \pm 9.18$ & 0.18 & 0.39 \\
\hline$B M I\left(\mathrm{~kg} / \mathrm{m}^{2}\right)$ & $23.7 \pm 4.77$ & $23.4 \pm 4.39$ & $24.5 \pm 5.60$ & 0.83 & 0.43 \\
\hline Waist circumference (cm) & $77.1 \pm 10.18$ & $77.0 \pm 9.29$ & $79.6 \pm 11.36$ & 0.77 & 0.17 \\
\hline$W H R$ & $0.78 \pm 0.052$ & $0.77 \pm 0.051$ & $0.78 \pm 0.054$ & 0.30 & 0.79 \\
\hline Abdominal circumference $(\mathrm{cm})$ & $86.3 \pm 10.21$ & $86.9 \pm 9.96$ & $88.7 \pm 11.69$ & 0.48 & 0.17 \\
\hline Gluteal circumference $(\mathrm{cm})$ & $98.7 \pm 9.51$ & $99.3 \pm 9.07$ & $101.4 \pm 10.66$ & 0.44 & 0.09 \\
\hline \multicolumn{6}{|l|}{ Biochemistry } \\
\hline Basal glycemia $(\mathrm{mmol} / \mathrm{l})$ & $4.86 \pm 0.519$ & $4.86 \pm 0.477$ & $4.82 \pm 0.486$ & 0.60 & 0.73 \\
\hline$A U C_{G l c 7}\left(\mathrm{mmol}^{*} \min / \mathrm{l}\right)$ & $1138 \pm 186.3$ & $1095 \pm 180.3$ & $1096 \pm 197.7$ & 0.06 & 0.13 \\
\hline Basal insulinemia $(\mathrm{mIU} / \mathrm{l})$ & $9.06 \pm 10.341$ & $6.54 \pm 3.767$ & $7.24 \pm 5.465$ & 0.24 & 0.34 \\
\hline$A U C_{I n s 7}\left(m I U^{*} \min / l\right)$ & $8097 \pm 6624.5$ & $6254 \pm 3132.9$ & $6841 \pm 4120.5$ & 0.002 & 0.04 \\
\hline Basal C-peptide (nmol/l) & $0.68 \pm 0.313$ & $0.62 \pm 0.206$ & $0.63 \pm 0.254$ & 0.65 & 0.44 \\
\hline$A U C_{C-p e p 7}\left(\mathrm{nmol}^{*} \min / \mathrm{l}\right)$ & $413.9 \pm 103.91$ & $365.3 \pm 98.44$ & $362.4 \pm 103.54$ & $<0.001$ & $<0.001$ \\
\hline HOMAF & $131.9 \pm 130.53$ & $101.1 \pm 60.24$ & $113.7 \pm 71.70$ & 0.03 & 0.43 \\
\hline$H O M A R$ & $2.04 \pm 2.421$ & $1.45 \pm 0.922$ & $1.60 \pm 1.340$ & 0.30 & 0.33 \\
\hline IS-Matsuda & $6.8 \pm 3.26$ & $8.0 \pm 3.77$ & $7.9 \pm 4.14$ & 0.02 & 0.09 \\
\hline IS-Cederholm & $58.1 \pm 65.02$ & $65.0 \pm 17.47$ & $63.7 \pm 17.78$ & 0.002 & 0.03 \\
\hline Cholesterol (mmol/l) & $4.6 \pm 0.87$ & $4.5 \pm 0.76$ & $4.5 \pm 0.74$ & 0.38 & 0.82 \\
\hline$H D L(\mathrm{mmol} / \mathrm{l})$ & $1.5 \pm 0.39$ & $1.6 \pm 0.39$ & $1.6 \pm 0.38$ & 0.04 & 0.21 \\
\hline$L D L(\mathrm{mmol} / \mathrm{l})$ & $2.6 \pm 0.77$ & $2.5 \pm 0.69$ & $2.5 \pm 0.73$ & 0.19 & 0.43 \\
\hline$T A G(\mathrm{mmol} / \mathrm{l})$ & $0.97 \pm 0.785$ & $0.85 \pm 0.465$ & $0.91 \pm 0.859$ & 0.57 & 0.57 \\
\hline Uric acid (umol/l) & $256.2 \pm 49.74$ & $258.4 \pm 54.25$ & $253.6 \pm 50.8$ & 0.50 & 0.81 \\
\hline$A L T(u k a t / l)$ & $0.34 \pm 0.178$ & $0.29 \pm 0.117$ & $0.32 \pm 0.188$ & 0.007 & 0.19 \\
\hline$A S T(u k a t / l)$ & $0.39 \pm 0.321$ & $0.35 \pm 0.130$ & $0.35 \pm 0.122$ & 0.14 & 0.11 \\
\hline GGT (ukat/l) & $0.28 \pm 0.152$ & $0.22 \pm 0.132$ & $0.29 \pm 0.424$ & 0.001 & 0.02 \\
\hline TSH (mIU/l) & $2.6 \pm 1.55$ & $2.8 \pm 2.70$ & $3.3 \pm 5.44$ & 0.57 & 0.55 \\
\hline fT3 (pmol/l) & $5.2 \pm 1.34$ & $5.0 \pm 1.47$ & $4.8 \pm 0.65$ & 0.23 & 0.02 \\
\hline fT4 (pmol/l) & $15.1 \pm 2.64$ & $15.7 \pm 3.92$ & $14.9 \pm 2.94$ & 0.51 & 0.84 \\
\hline
\end{tabular}

Values are given as mean $\pm S D$, p-level according to Mann-Whitney test.

Association of birth weight with biochemical and anthropometric data

In this study, birth weight was systematically associated with glucose processing.

Results in the GDM group are listed in Table 2: All 376 women with history of gestational diabetes were categorized according to their birth weight quartile to the three groups referred to as low, medium, and high birth weight category. These groups did not differ in age, so that there was no need to adjust for age. Significantly higher stimulated insulinemia and C-peptide levels were observed in low birth weight category compared with medium and high birth weight subgroups. For more details concerning insulin and C-peptide concentrations in the particular minutes during the oGTT see Figure 1a. Consequently, indices of insulin sensitivity were lower in low birth weight women compared to medium and high birth weight subgroup, see Table 2. Repeated 
a

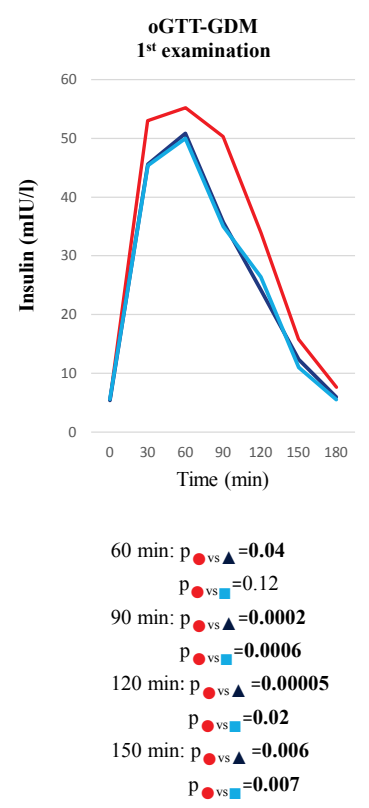

b

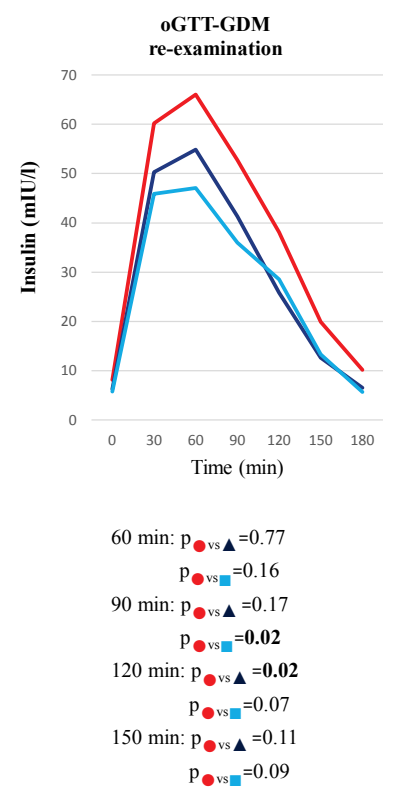

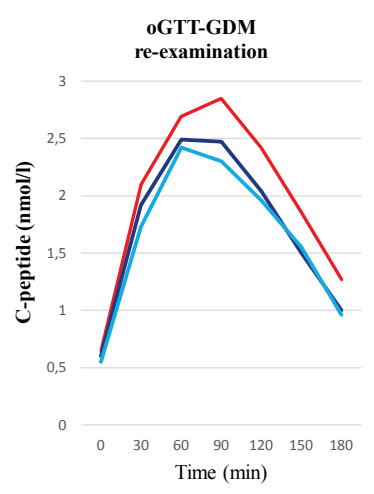

60 min: $\mathrm{p}_{\text {vs }} \Delta=0.19$

$$
\mathrm{p}_{\text {○vs }}=0.07
$$$$
90 \text { min: } \mathrm{p}_{\text {vs }} \mathbf{\Lambda}=0.18
$$$$
\mathrm{p}_{\text {v } \mathrm{vs}}=\mathbf{0 . 0 4}
$$$$
120 \text { min: } \mathrm{p}_{\bullet \mathrm{vs}} \mathbf{= 0 . 0 4}
$$$$
\mathrm{p}_{\text {○vs }}=\mathbf{0 . 0 3}
$$$$
150 \text { min: } \mathrm{p}_{\bullet \mathrm{vs}}=0.12
$$$$
\mathrm{p}_{\text {○ vs }}=0.06
$$

Fig. 1. a) Response to glucose in GDM group ( $n=376)$ ( $p$-level according to Mann-Whitney test), b) Response to glucose in re-examined GDM group after 5-8 years $(n=107)$ ( $p$-level according to Mann-Whitney test).

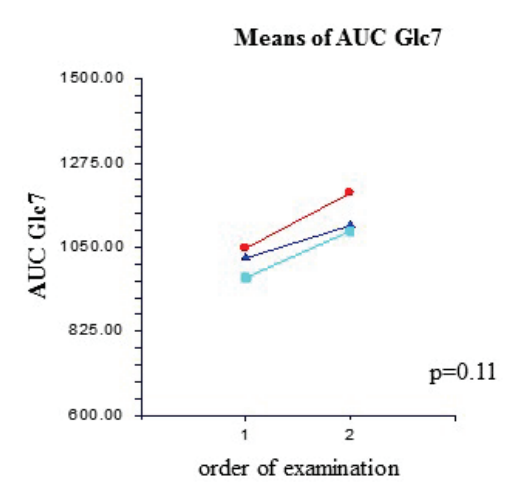

Means of HOMAR

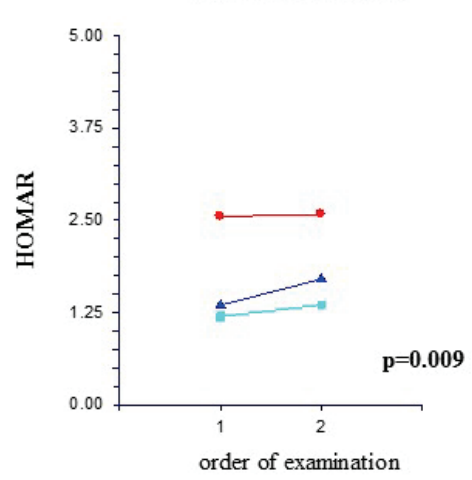

Means of AUC I7

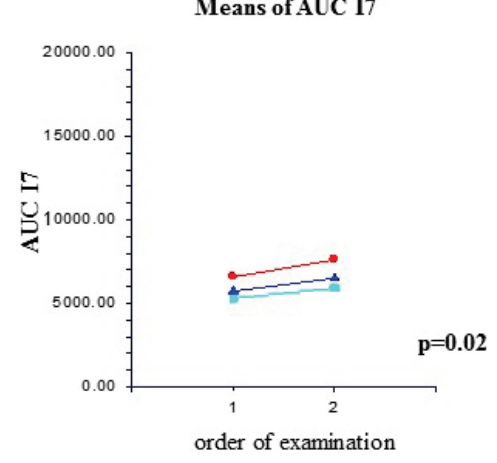

Means of IS-Matsuda

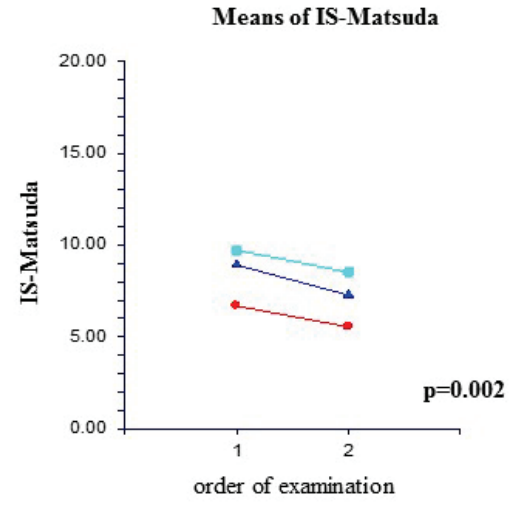

Means of AUC C-pep7

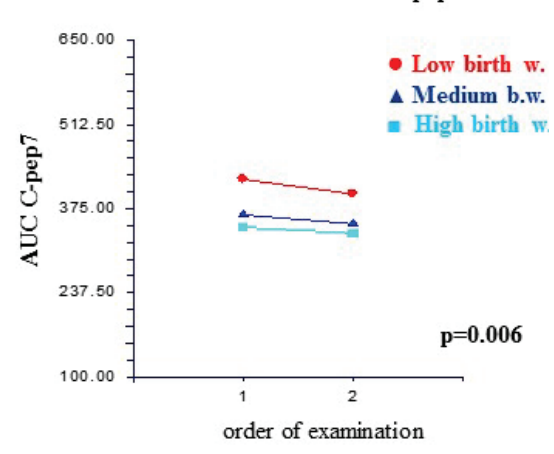

Means of IS-Cederholm

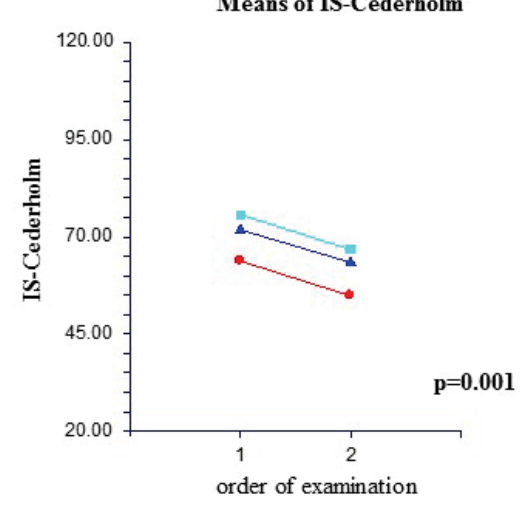

Fig. 2. Re-examined GDM group ( $n=107$ ) - birth weight factor in glucose metabolism (Repeated Measures ANOVA).

examinations in the GDM group after 5-8 years indicate that association of low birth weight with insulin secretion persists, for details see Figure 1b. Repeated measures
ANOVA analysis, evaluating metabolic changes of each individual over time separately, confirmed birth weight as a significant factor for glucose metabolism in gestational 
Table 3. Differences between birth weight categories in control subjects.

\begin{tabular}{|c|c|c|c|c|c|}
\hline & $\begin{array}{l}\text { 1. quartile } \\
\leq 2900 \mathrm{~g}\end{array}$ & $\begin{array}{c}\text { 2. + 3. quartile } \\
>2900-\leq 3500 \mathrm{~g}\end{array}$ & $\begin{array}{l}\text { 4. quartile } \\
>3500 \mathrm{~g}\end{array}$ & $\begin{array}{c}\text { p } \\
\text { 1. vs } 2 .+3 \text {. } \\
\text { quartile }\end{array}$ & $\begin{array}{c}\text { p } \\
\text { 1. vs } 4 . \\
\text { quartile }\end{array}$ \\
\hline$n(\Sigma=80)$ & 19 & 43 & 18 & - & - \\
\hline Age (years) & $44.3 \pm 9.53$ & $42.2 \pm 9.36$ & $40.4 \pm 8.97$ & 0.47 & 0.13 \\
\hline \multicolumn{6}{|l|}{ Anthropometry } \\
\hline Systolic blood pressure & $120.6 \pm 12.85$ & $116.8 \pm 13.50$ & $116.9 \pm 19.42$ & 0.20 & 0.46 \\
\hline Diastolic blood pressure & $76.7 \pm 10.77$ & $73.9 \pm 9.26$ & $76.4 \pm 11.08$ & 0.33 & 0.93 \\
\hline$B M I\left(\mathrm{~kg} / \mathrm{m}^{2}\right)$ & $24.4 \pm 4.44$ & $24.6 \pm 5.09$ & $25.2 \pm 4.40$ & 0.93 & 0.51 \\
\hline Waist circumference (cm) & $77.7 \pm 11.74$ & $77.9 \pm 10.08$ & $77.8 \pm 10.54$ & 0.74 & 0.81 \\
\hline$W H R$ & $0.77 \pm 0.077$ & $0.76 \pm 0.044$ & $0.74 \pm 0.049$ & 1.00 & 0.37 \\
\hline Abdominal circumference $(\mathrm{cm})$ & $84.0 \pm 10.99$ & $86.7 \pm 11.61$ & $87.7 \pm 12.64$ & 0.38 & 0.34 \\
\hline Gluteal circumference $(\mathrm{cm})$ & $100.0 \pm 7.15$ & $102.1 \pm 9.68$ & $104.7 \pm 11.84$ & 0.44 & 0.24 \\
\hline \multicolumn{6}{|l|}{ Biochemistry } \\
\hline Basal glycemia $(\mathrm{mmol} / \mathrm{l})$ & $4.72 \pm 0.530$ & $4.97 \pm 0.759$ & $4.69 \pm 0.371$ & 0.27 & 0.89 \\
\hline$A U C_{G l c 7}\left(\mathrm{mmol}^{*} \min / \mathrm{l}\right)$ & $1089 \pm 222.1$ & $1075 \pm 310.5$ & $946 \pm 129.0$ & 0.75 & 0.04 \\
\hline Basal insulinemia $(\mathrm{mIU} / \mathrm{l})$ & $7.12 \pm 5.097$ & $7.59 \pm 5.721$ & $7.11 \pm 5.174$ & 0.64 & 0.71 \\
\hline$A U C_{I n s 7}\left(m I U^{*} \min / l\right)$ & $6725 \pm 4919.4$ & $6327 \pm 5095.9$ & $5572 \pm 3896.7$ & 0.55 & 0.38 \\
\hline Basal C-peptide (nmol/l) & $0.62 \pm 0.318$ & $0.63 \pm 0.251$ & $0.56 \pm 0.240$ & 0.32 & 0.73 \\
\hline$A U C_{C-p e p 7}\left(\mathrm{nmol}^{*} \min / \mathrm{l}\right)$ & $405.2 \pm 145.19$ & $395.5 \pm 134.55$ & $325.1 \pm 99.23$ & 0.99 & 0.11 \\
\hline HOMAF & $110.4 \pm 60.77$ & $108.9 \pm 60.28$ & $125.1 \pm 72.79$ & 0.88 & 0.62 \\
\hline$H O M A R$ & $1.57 \pm 1.334$ & $1.76 \pm 1.600$ & $1.51 \pm 1.227$ & 0.58 & 0.79 \\
\hline IS-Matsuda & $8.9 \pm 5.23$ & $8.4 \pm 4.33$ & $9.0 \pm 3.53$ & 0.92 & 0.68 \\
\hline IS-Cederholm & $66.7 \pm 21.61$ & $71.2 \pm 23.45$ & $79.7 \pm 19.08$ & 0.57 & 0.08 \\
\hline Cholesterol (mmol/l) & $4.8 \pm 0.92$ & $4.8 \pm 0.77$ & $4.8 \pm 0.65$ & 0.97 & 0.90 \\
\hline$H D L(\mathrm{mmol} / \mathrm{l})$ & $1.6 \pm 0.31$ & $1.5 \pm 0.30$ & $1.6 \pm 0.42$ & 0.37 & 0.85 \\
\hline$L D L(\mathrm{mmol} / \mathrm{l})$ & $2.7 \pm 0.87$ & $2.8 \pm 0.69$ & $2.8 \pm 0.73$ & 0.61 & 0.94 \\
\hline$T A G(\mathrm{mmol} / \mathrm{l})$ & $1.20 \pm 0.584$ & $1.04 \pm 0.492$ & $0.95 \pm 0.442$ & 0.26 & 0.16 \\
\hline Uric acid (umol/l) & $242.8 \pm 49.85$ & $232.7 \pm 66.48$ & $248.1 \pm 49.35$ & 0.40 & 0.93 \\
\hline$A L T(u k a t / l)$ & $0.39 \pm 0.194$ & $0.38 \pm 0.279$ & $0.30 \pm 0.109$ & 0.52 & 0.14 \\
\hline AST (ukat/l) & $0.47 \pm 0.217$ & $0.38 \pm 0.142$ & $0.36 \pm 0.117$ & 0.09 & 0.08 \\
\hline GGT (ukat/l) & $0.39 \pm 0.404$ & $0.31 \pm 0.277$ & $0.22 \pm 0.115$ & 0.76 & 0.13 \\
\hline TSH (mIU/l) & $2.5 \pm 1.83$ & $2.4 \pm 1.50$ & $2.5 \pm 1.44$ & 0.87 & 0.59 \\
\hline fT3 (pmol/l) & $4.9 \pm 0.80$ & $4.8 \pm 0.99$ & $5.5 \pm 2.58$ & 0.59 & 0.88 \\
\hline fT4 (pmol/l) & $15.2 \pm 2.20$ & $15.5 \pm 2.46$ & $16.3 \pm 5.41$ & 0.49 & 0.58 \\
\hline
\end{tabular}

Values are given as mean $\pm \mathrm{SD}$, p-level according to Mann-Whitney test.

diabetics, see Figure 2. No significant differences between the birth weight categories were observed in anthropometric parameters, blood pressure, or uric acid. Subtle differences in lipid spectra, hepatic enzymes, thyroid hormones, and all details concerning differences between birth weight categories in gestational diabetics are summarized in Table 2.

Results in the control group are specified in
Table 3: Analogously to gestational diabetics, controls were devided to the three groups according to birth weight categories, these groups also did not differ in age. Relationship between low birth weight and glucose metabolism was expressed by higher postchallenge glycemia during oGTT when compared with high birth weight group, but not by higher insulin secretion or lower indices of insulin sensitivity. We did not observe any 
relation of birth weight to other tested biochemical and anthropometric components of MetS.

\section{Discussion}

Much of the currently available knowledge on the consequences of birth weight and metabolic risks throughout the life course has been provided by studies based on inconsistent criteria in terms of how to define low or high birth weight. In numerically abundant studies, low birth weight is frequently considered to be $<2.5 \mathrm{~kg}$ or $<10^{\text {th }}$ population-based percentile (Malin et al. 2015). As numbers of our participants were limited, we defined low birth weight subjects as stated in methods.

Our study did not proved that low birth weight is a risk factor for GDM development, but it shows systematic and long-term association between low birth weight and glucose processing in the group of women with positive history of GDM. Low birth weight GDM women reveal significantly higher postchallenge insulin secretion while postchallenge glycemia remains comparable with medium and high birth weight GDM group. This condition is reflected in lower indices of peripheral insulin sensitivity. The adverse state persists more than five years after delivery.

The results described are notable taking into account fetal insulin hypothesis (Hattersley and Tooke 1999). According to this theory, lower birth weight is a reflection of lower prenatal insulin secretion, as insulin represents key fetal growth factor. In our study, women born with low birth weight on the contrary demonstrate in the adulthood higher postchallenge insulin secretion, which is well documented also by C-peptide levels. One possible viewpoint could be based on fetal programming hypothesis postulating that early life events play a powerful role in influencing health condition throughout lifespan. Low birth weight may reflect low intrauterine demands for fetal insulin secretion. Low insulin demands correspond with lower glucose delivery by mother and thereby with lower birth weight (Breschi et al. 1993). These early transient environmental conditions in a crucial period for establishing metabolic pathways mediated by tiny shift of the hypothalamic- pituitary-adrenal axis set point (Clark et al. 1998) and/or epigenetic modifications (Heijmans et al. 2008) may affect glucose sensing and subsequent insulin response in late adulthood. Our observations indicate that lower exposition to glucose in utero coupled with low fetal insulin response resulting in low birth weight may in adults lead to higher insulin demands when exposed to high glucose load in order to restore and maintain normal glycemia.

Another possible explanation for the obtained data corresponds well with conclusive results in gestational diabetics. We cannot be sure, that low birth weight children are small due to low insulin secretion. They can be small also because their response to insulin, including insulin-mediated growth rate, is lower. Prenatally initiated reduced sensitivity towards insulin can persist into adulthood and, in pregnancy, it may contribute to development of GDM.

Of course, all the mentioned relations are highly dependent on genetic and epigenetic background, since both birth weight and insulin sensitivity are to a great extent modulated by genes in their functional plasticity.

In conclusion, our findings suggest that low birth weight and positive GDM history are synergistically linked with glucose metabolism and peripheral insulin sensitivity in adulthood, in particular via postchallenge insulin secretion. These observations highlight the importance of birth weight as an integral part of medical history record, especially in people at increased diabetes risk.

\section{Conflict of Interest}

There is no conflict of interest.

\section{Acknowledgements}

Grant support IGA MH CR NT/13544-4/2012.

\begin{abstract}
Abbreviations
AUC, area under the curve; BMI, body mass index; GDM, gestational diabetes mellitus; HOMA, homeostasis models assessment; HDL, high density lipoprotein; LDL, low density lipoprotein; MetS, metabolic syndrome; oGTT, oral glucose-tolerance test; T2DM, type 2 diabetes mellitus; TAG, triacylglycerols; WHR, waist to hip ratio.
\end{abstract}

\section{References}

ANDĚLOVÁ K: Recommended practice for screening of impaired glucose tolerance during pregnancy (in Czech). Čes Gynekol 74: 7, 2009. 
BABENKO AP, POLAK M, CAVÉ H, BUSIAH K, CZERNICHOW P, SCHARFMANN R, BRYAN J, AGUILARBRYAN L, VAXILLAIRE M, FROGUEL P: Activating mutations in the ABCC8 gene in neonatal diabetes mellitus. N Engl J Med 355: 456-466, 2006.

BARKER DJ, HALES CN, FALL CH, OSMOND C, PHIPPS K, CLARK PM: Type 2 (non-insulin-dependent) diabetes mellitus, hypertension and hyperlipidaemia (syndrome $\mathrm{X}$ ): relation to reduced fetal growth. Diabetologia 36: 62-67, 1993.

BRESCHI MC, SEGHIERI G, BARTOLOMEI G, GIRONI A, BALDI S, FERRANNINI E: Relation of birthweight to maternal plasma glucose and insulin concentrations during normal pregnancy. Diabetologia 36: 1315-1321, 1993.

CLARK PM: Programming of the hypothalamo-pituitary-adrenal axis and the fetal origins of adult disease hypothesis. Eur J Pediatr 157: 7-10, 1998.

DABELEA D, HANSON RL, LINDSAY RS, PETTITT DJ, IMPERATORE G, GABIR MM, ROUMAIN J, BENNETT PH, KNOWLER WC: Intrauterine exposure to diabetes conveys risks for type 2 diabetes and obesity: a study of discordant sibships. Diabetes 49: 2208-2211, 2000.

DEBOER MD, DONG L, GURKA MJ: Racial/ethnic and sex differences in the relationship between uric acid and metabolic syndrome in adolescents: an analysis of National Health and Nutrition Survey 1999-2006. Metabolism 61: 554-561, 2012.

DURMUŞ B, MOOK-KANAMORI DO, HOLZHAUER S, HOFMAN A, VAN DER BEEK EM, BOEHM G, STEEGERS EA, JADDOE VW: Growth in foetal life and infancy is associated with abdominal adiposity at the age of 2 years: the generation R study. Clin Endocrinol (Oxf) 72: 633-640, 2010.

EDGHILL EL, BINGHAM C, SLINGERLAND AS, MINTON JA, NOORDAM C, ELLARD S, HATTERSLEY AT: Hepatocyte nuclear factor-1 beta mutations cause neonatal diabetes and intrauterine growth retardation: support for a critical role of HNF-1beta in human pancreatic development. Diabet Med 23: 1301-1306, 2006.

ERIKSSON JG, FORSEN TJ, OSMOND C, BARKER DJ: Pathways of infant and childhood growth that lead to type 2 diabetes. Diabetes Care 26: 3006-3010, 2003.

FOWDEN AL, FORHEAD AJ: Endocrine interactions in the control of fetal growth. Nestle Nutr Inst Workshop Ser 74: 91-102, 2013.

FRAYLING TM, HATTERSLEY AT: The role of genetic susceptibility in the association of low birth weight with type 2 diabetes. Br Med Bull 60: 89-101, 2001.

FREATHY RM, WEEDON MN, BENNETT A, HYPPONEN E, RELTON CL, KNIGHT B, SHIELDS B, PARNELL KS, GROVES CJ, RING SM, PEMBREY ME, BEN-SHLOMO Y, STRACHAN DP, POWER C, JARVELIN MR, MCCARTHY MI, DAVEY SMITH G, HATTERSLEY AT, FRAYLING TM: Type 2 diabetes TCF7L2 risk genotypes alter birth weight: a study of 24,053 individuals. Am J Hum Genet 80: 1150-1161, 2007.

FREEMAN MS, MANSFIELD MW, BARRETT JH, GRANT PJ: Heritability of features of the insulin resistance syndrome in a community-based study of healthy families. Diabet Med 19: 994-999, 2002.

GODFREY KM, BARKER DJ: Fetal nutrition and adult disease. Am J Clin Nutr 71: 1344S-1352S, 2000.

HALES CN, BARKER DJ, CLARK PM, COX LJ, FALL C, OSMOND C, WINTER PD: Fetal and infant growth and impaired glucose tolerance at age 64. BMJ 303: 1019-1022, 1991.

HARDER T, RODEKAMP E, SCHELLONG K, DUDENHAUSEN JW, PLAGEMANN A: Birth weight and subsequent risk of type 2 diabetes: a meta-analysis. Am J Epidemiol 165: 849-857, 2007.

HATTERSLEY AT, TOOKE JE: The fetal insulin hypothesis: an alternative explanation of the association of low birthweight with diabetes and vascular disease. Lancet 353: 1789-1792, 1999.

HATTERSLEY AT, BEARDS F, BALLANTYNE E, APPLETON M, HARVEY R, ELLARD S: Mutations in the glucokinase gene of the fetus result in reduced birth weight. Nat Genet 19: 268-270, 1998.

HEIJMANS BT, TOBI EW, STEIN AD, PUTTER H, BLAUW GJ, SUSSER ES, SLAGBOOM PE, LUMEY LH: Persistent epigenetic differences associated with prenatal exposure to famine in humans. Proc Natl Acad Sci USA 105: 17046-17049, 2008.

IBÁÑEZ L, ONG K, DE ZEGHER F, MARCOS MV, DEL RIO L, DUNGER DB: Fat distribution in non-obese girls with and without precocious pubarche: central adiposity related to insulinaemia and androgenaemia from prepuberty to postmenarche. Clin Endocrinol (Oxf) 58: 372-379, 2003. 
KALRA S, UNNIKRISHNAN AG: Obesity in India: the weight of the nation. $J$ Med Nutr Nutraceut 1: 37-41, 2012.

KITSANTAS P, GAFFNEY KF: Risk profiles for overweight/obesity among preschoolers. Early Hum Dev 86: 563-568, 2010.

MALIN GL, MORRIS RK, RILEY RD, TEUNE MJ, KHAN KS: When is birthweight at term ( $\geq 37$ weeks' gestation) abnormally low? A systematic review and meta-analysis of the prognostic and predictive ability of current birthweight standards for childhood and adult outcomes. BJOG 122: 634-642, 2015.

MEAS T, DEGHMOUN S, ALBERTI C, CARREIRA E, ARMOOGUM P, CHEVENNE D, LÉVY-MARCHAL C: Independent effects of weight gain and fetal programming on metabolic complications in adults born small for gestational age. Diabetologia 53: 907-913, 2010.

MILLS GW, AVERY PJ, MCCARTHY MI, HATTERSLEY AT, LEVY JC, HITMAN GA, SAMPSON M, WALKER M: Heritability estimates for beta cell function and features of the insulin resistance syndrome in UK families with an increased susceptibility to type 2 diabetes. Diabetologia 47: 732-738, 2004.

MITANCHEZ D, YZYDORCZYK C, SIDDEEK B, BOUBRED F, BENAHMED M, SIMEONI U: The offspring of the diabetic mother - short- and long-term implications. Best Pract Res Clin Obstet Gynaecol 29: 256-269, 2015.

MOHAN V, JAYDIP R, DEEPA R: Type 2 diabetes in Asian Indian youth. Pediatr Diabetes 8: 28-34, 2007.

OLDROYD J, RENZAHO A, SKOUTERIS H: Low and high birth weight as risk factors for obesity among 4 to 5 -yearold Australian children: does gender matter? Eur J Pediatr 170: 899-906, 2011.

ONG KK, DUNGER DB: Thrifty genotypes and phenotypes in the pathogenesis of type 2 diabetes mellitus. $J$ Pediatr Endocrinol Metab 13: 1419-1424, 2000.

ONG KK, AHMED ML, EMMETT PM, PREECE MA, DUNGER DB: Association between postnatal catch-up growth and obesity in childhood: prospective cohort study. BMJ 320: 967-971, 2000.

PULIZZI N, LYSSENKO V, JONSSON A, OSMOND C, LAAKSO M, KAJANTIE E, BARKER DJ, GROOP LC, ERIKSSON JG: Interaction between prenatal growth and high-risk genotypes in the development of type 2 diabetes. Diabetologia 52: 825-829, 2009.

SLINGERLAND AS, HATTERSLEY AT: Activating mutations in the gene encoding Kir6.2 alter fetal and postnatal growth and also cause neonatal diabetes. J Clin Endocrinol Metab 91: 2782-2788, 2006.

SNIEDER H, BOOMSMA DI, VAN DOORNEN LJ, NEALE MC: Bivariate genetic analysis of fasting insulin and glucose levels. Genet Epidemiol 16: 426-446, 1999.

STETTLER N, BOVET P, SHAMLAYE H, ZEMEL BS, STALLINGS VA, PACCAUD F: Prevalence and risk factors for overweight and obesity in children from Seychelles, a country in rapid transition: the importance of early growth. Int J Obes Relat Metab Disord 26: 214-219, 2002a.

STETTLER N, ZEMEL BS, KUMANYIKA S, STALLINGS VA: Infant weight gain and childhood overweight status in a multicenter, cohort study. Pediatrics 109: 194-199, 2002 b.

STØY J, EDGHILL EL, FLANAGAN SE, YE H, PAZ VP, PLUZHNIKOV A, BELOW JE, HAYES MG, COX NJ, LIPKIND GM, LIPTON RB, GREELEY SA, PATCH AM, ELLARD S, STEINER DF, HATTERSLEY AT, PHILIPSON LH, BELL GI: Insulin gene mutations as a cause of permanent neonatal diabetes. Proc Natl Acad Sci USA 104: 15040-15044, 2007.

TZOULAKI I, SOVIO U, PILLAS D, HARTIKAINEN AL, POUTA A, LAITINEN J, TAMMELIN TH, JARVELIN MR, ELLIOTT P: Relation of immediate postnatal growth with obesity and related metabolic risk factors in adulthood: the northern Finland birth cohort 1966 study. Am J Epidemiol 171: 989-998, 2010.

VELASQUEZ-MIEYER P, PEREZ-FAUSTINELLI S, COWAN PA: Identifying children at risk for obesity, type 2 diabetes, and cardiovascular disease. Diabetes Spectrum 18: 213-220, 2005.

WEEDON MN, CLARK VJ, QIAN Y, BEN-SHLOMO Y, TIMPSON N, EBRAHIM S, LAWLOR DA, PEMBREY ME, RING S, WILKIN TJ, VOSS LD, JEFFERY AN, METCALF B, FERRUCCI L, CORSI AM, MURRAY A, MELZER D, KNIGHT B, SHIELDS B, SMITH GD, HATTERSLEY AT, DI RIENZO A, FRAYLING TM: A common haplotype of the glucokinase gene alters fasting glucose and birth weight: association in six studies and population-genetics analyses. Am J Hum Genet 79: 991-1001, 2006. 
WHINCUP PH, KAYE SJ, OWEN CG, HUXLEY R, COOK DG, ANAZAWA S, BARRETT-CONNOR E, BHARGAVA SK, BIRGISDOTTIR BE, CARLSSON S, DE ROOIJ SR, DYCK RF, ERIKSSON JG, FALKNER B, FALL C, FORSÉN T, GRILL V, GUDNASON V, HULMAN S, HYPPÖNEN E, JEFFREYS M, LAWLOR DA, LEON DA, MINAMI J, MISHRA G, OSMOND C, POWER C, RICH-EDWARDS JW, ROSEBOOM TJ, SACHDEV HS, SYDDALL H, THORSDOTTIR I, VANHALA M, WADSWORTH M, YARBROUGH DE: Birth weight and risk of type 2 diabetes: a systematic review. JAMA 300: 2886-2897, 2008.

YAJNIK C: Interactions of perturbations in intrauterine growth and growth during childhood on the risk of adult-onset disease. Proc Nutr Soc 59: 257-265, 2000. 